\title{
Studies of RPC detector operation with eco-friendly gas mixtures under irradiation at the CERN Gamma Irradiation Facility
}

\author{
Gianluca Rigoletti $^{* n n}$, G. Aielli ${ }^{b}$ G. Alberghi $^{c}$ L. Benussi $^{d}$ A. Bianchi ${ }^{k}$ S. Bianco ${ }^{d}$ D. \\ Boscherini $^{c}$ A. Bruni ${ }^{c}$ P. Camarri ${ }^{b}$ R. Cardarelli ${ }^{a}$ M. Corbetta ${ }^{m n}$ S. Delsanto ${ }^{k}$ A. Di \\ Ciaccio $^{b}$ L. Di Stante ${ }^{b}$ P. Dupieux ${ }^{l}$ J. Eysermans ${ }^{j}$ A. Ferretti ${ }^{k}$ M. Ferrini $^{e}$ M. \\ Gagliardi $^{k}$ A. Gelmi ${ }^{g}$ R. Guida ${ }^{n}$ B. Joly ${ }^{l}$ B. Liberti ${ }^{a}$ B. Mandelli ${ }^{n}$, S.P. Manen ${ }^{l}$ L. \\ Micheletti $^{k}$ L. Passamonti ${ }^{d}$ E. Pastori ${ }^{a}$ D. Piccolo ${ }^{d}$ D. Pierluigi ${ }^{d}$ A. Polini ${ }^{c}$ G. \\ Pugliese $^{g}$ L. Quaglia ${ }^{k}$ A. Russo ${ }^{d}$ P. Salvini ${ }^{h}$ R. Santonico ${ }^{b}$ G. Saviano ${ }^{e}$ L. Terlizzi ${ }^{k}$ M. \\ Tytgat $^{i}$ E. Vercellin ${ }^{k}$ N. Zaganidis ${ }^{i}$ \\ ${ }^{a}$ INFN, Tor Vergata, Rome, Italy \\ ${ }^{b}$ Dipartimento di Fisica di Roma Tor Vergata, Rome, Italy \\ ${ }^{c}$ INFN, Bologna \\ ${ }^{d}$ Laboratori Nazionali di Frascati dell'INFN \\ e Sapienza Universita di Roma, Rome, Italy \\ ${ }^{f}$ Laboratori Nazionali di Frascati dell'INFN \\ ${ }^{g}$ Dipartimento di Fisica di Bari e sezione INFN di Bari \\ ${ }^{h}$ Sezione INFN di Pavia \\ ${ }^{i}$ Ghent University, Dept. of Physics and Astronomy, Ghent, Belgium \\ ${ }^{j}$ Benemérita Universidad Autónoma de Puebla \\ ${ }^{k}$ Universitá degli Studi di Torino and INFN, Torino, Italy \\ ${ }^{l}$ Clermont Université, Université Blaise Pascal, Clermont-Ferrand, France \\ ${ }^{m}$ Université Claude Bernard Lyon I, Lyon, France \\ ${ }^{n}$ CERN, Geneve, Switzerland \\ E-mail: gianluca.rigoletti@cern.ch
}


Resistive Plate Chamber (RPC) detectors are widely used at the CERN LHC experiments as muon trigger thanks to their excellent time resolution. They are operated with a Freon-based gas mixture containing $\mathrm{C}_{2} \mathrm{H}_{2} \mathrm{~F}_{4}$ and $\mathrm{SF}_{6}$, both greenhouse gases (GHG) with a very high global warming potential (GWP). The search of new environmentally friendly gas mixtures is necessary to reduce GHG emissions and costs as well as to optimize RPC performance. Several recently available gases with low GWP have been identified as possible replacements for $\mathrm{C}_{2} \mathrm{H}_{2} F_{4}$ and $S F_{6}$. In particular, eco-friendly gas mixtures based on the HFO-1234ze have been investigated on 1.4 and 2 mm single-gap and double-gap RPCs. The RPC detectors have been tested at the CERN Gamma Irradiation Facility (GIF++), which provides a high energy muon beam combined with an intense gamma source allowing to simulate the background expected at HL-LHC. The performance of RPCs were studied at different gamma rates with the new environmentally friendly gases by measuring ohmic and physics currents, fluorine radicals and HF production, rate capability and induced charge. Preliminary results on the long-term effects on the performance of the detectors are presented in this study.

European Physical Society Conference on High Energy Physics - EPS-HEP2019 10-17 July, 2019

Ghent, Belgium

${ }^{*}$ Speaker. 


\section{Introduction}

The Resistive Plate Chambers (RPC) are gaseous particle detectors [1], employed at the CERNLHC experiments thanks to their time resolution and production costs. They are used at the ATLAS, CMS and ALICE experiments for the muon trigger systems. The detectors are operated with a large fraction (between $90 \%$ and $95 \%$ ) of $\mathrm{C}_{2} \mathrm{H}_{2} \mathrm{~F}_{4}$ known commercially as R-134a. In addition, $4.5 \% i_{4} H_{10}$ and $0.3 \%$ of $S F_{6}$ is used to operate the RPCs in avalanche mode. Both R-134a and the $S F_{6}$ are known to be Greenhouse Gases (GHG) with a Global Warming Potential (GWP) of 1430 and 22800 respectively. Despite the use of gas recirculation systems at the three experiments, the ATLAS and CMS RPC systems account for the 87\% of the CERN GHG emissions due to the presence of leaks at detectors level [2] ${ }^{1}$. Starting from January 2015 the European Union defined a set of regulations [3] aiming at reducing the GHG emissions from fluorinated gases. The strategies defined can be summarized in four main points:

- Restrict the placing on the market by reducing products availability of fluorinated GHGs.

- Ban the use of GHGs where eco-friendly alternatives are already available.

- Require regular and certified check controls on leaks for existing equipments.

- Require a recovery of the gases at the end of the equipments life.

The search for eco-friendly gas mixtures for RPCs operation plays therefore a central role in the reduction of the GHG emissions and costs. Furthermore, the search for new gas mixtures may be advisable for performance improvement of the detectors and gas systems operation.

\subsection{The ECOGAS collaboration}

The ECOGAS collaboration was born as a joint effort of the main LHC expeirments together with the CERN gas team to study the HFO properties and search for HFO based gas mixtures suitable for RPC detectors operations. The ECOGAS collaboration consists of the participation of the ALICE, ATLAS, CMS and CERN Gas team.

\section{HFO-1234ze as a replacements of $\mathrm{R}-134 \mathrm{a}$}

The search for new eco-friendly gas mixtures is particularly challenging for LHC systems as several requirements must be fulfilled for the gas components to be suitable for the correct operation of the detectors [4]. In particular, the gas mixture at the ATLAS and CMS experiments requires that:

- The gas mixture must not be harmful for the human health, meaning that the mixture should be of zero or low toxicity.

- The gas mixture must not be flammable or explosive in the atmosphere, although some components taken alone may be flammable. In the ATLAS/CMS gas mixture some Isobutane is present in such a quantity that it improves the detector operation while keeping the gas mixture incombustible.

\footnotetext{
${ }^{1} \mathrm{~A}$ campaign of leaks reparation was scheduled for the LS2 phase and is currently going on
} 
- The detector working point should be compatible with the front-end electronics and power supply systems that are currently being used.

A low-GWP gas in the family of the Hydro-Fluoro-Olefin (HFO) named HFO1234-ze was developed as a possible replacement of R-134a in refrigerant applications. HFO1234-ze has a GWP of 6 and falls within the category of low-flammability and low-toxicity gases. An addition of a gas such as $\mathrm{CO}_{2}$, is required to keep the working point lower than $12 \mathrm{kV}$. A small amount of Isobutane and $S F_{6}$ is still required as their properties are necessary to reduce photon-feedback effect, limiting the streamers pulses and ensuring the desired rate capability.

\section{Setup}

\subsection{The Gamma Irradiation Facility (GIF++)}

To conduct the study under LHC-like radiation the setup was installed at the CERN Gamma Irradiation Facility (GIF++) [5]. The GIF++ is located at CERN a ${ }^{137} \mathrm{Cs}$ of $14 \mathrm{TBq}$ is installed inside, giving the possibility to simulate the conditions foreseen during the High Luminosity LHC (HL-LHC). A system of absorption filters (ABSs) made out of lead can be used to adjust the intensity of the radiation, i.e. the rate of photons impinging the detectors.

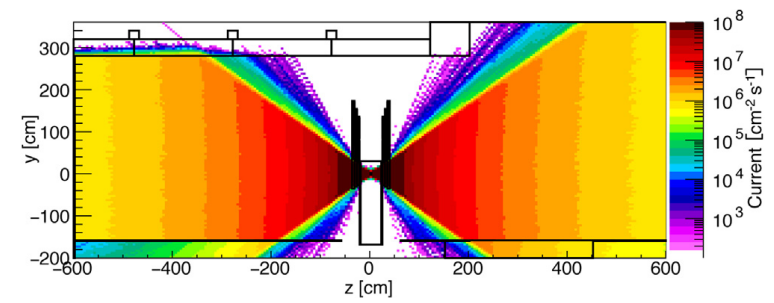

Figure 1: Irradiation field of the GIF++ [5]

\subsection{Experimental Setup}

For the present study a dedicated setup was used. The setup can be divided into three main parts: the detectors together with the mechanical frame inside the GIF++ bunker; the power supply systems and electronics readout; the gas supply system. The detectors were built out of High Pressure Laminate (HPL), commonly referred to as bakelite. Table 1 shows the RPCs dimensions and gap types used for the experimental setup. All the chambers were installed in vertical position, facing the source, on a single mechanical support frame.

A dedicated gas mixer, located in the gas service room, was used to send the gas mixture to the detectors. A humidifier module was also installed to keep the gas mixture humidified at $40 \%$ of relative humidity to ensure a stable resistivity value of the electrodes [6]. The gas was then sent in the bunker and the main input line was split into several different lines, one for each chamber.

\section{Characterization of the gas mixture}

The detectors were first operated using the ATLAS and CMS gas mixture (also referred to as standard gas mixture in the following) and the performance were compared against a mixture 


\begin{tabular}{lllll}
\hline Chamber name & Gap $[\mathrm{mm}]$ & Thickness $[\mathrm{mm}]$ & Type & Area $\left[\mathrm{cm}^{2}\right]$ \\
\hline CMS-RPC & 2 & 2 & Double gap & 7000 \\
CMS-iRPC & 1.4 & 1.4 & Double gap & 7000 \\
ATLAS-2-0 & 2 & 2 & Single gap & 500 \\
ALICE-2-0 & 2 & 2 & Single gap & 2500 \\
EPDT-RPC3 & 2 & 2 & Single gap & 7000 \\
\hline
\end{tabular}

Table 1: Detectors used for the experimental setup

of $\mathrm{HFO} / \mathrm{CO}_{2} / \mathrm{iC}_{4} \mathrm{H}_{10} / \mathrm{SF}_{6} 45 / 50 / 4 / 1$, referred to also as ecogas mixture. Figure 2 (a) shows that at fixed background rate the knee of the ecogas mixture is $1.8 \mathrm{kV}$ higher than the one of the standard gas mixture.

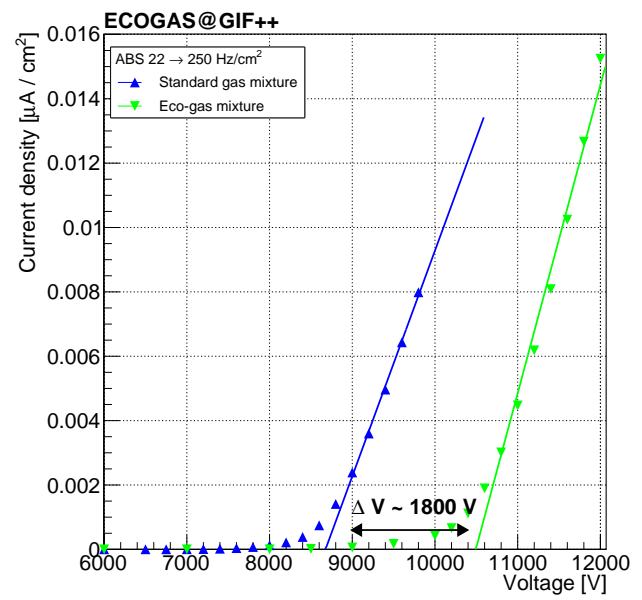

(a) Current scan for the EP-DT chamber with same irradiation conditions and different gas mixtures. The difference in the knee is $1.8 \mathrm{kV}$

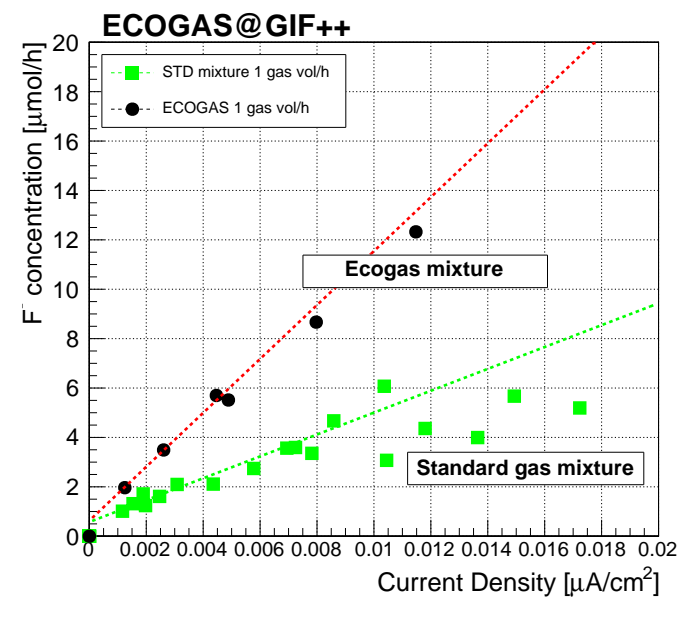

(b) Rate of $F^{-}$production for the CMS-GT chamber with the same flow per volume and different gas mixtures.

Figure 2: Comparison of the Standard Gas Mixture against the ECOGAS mixture

A measure of the accumulation of fluorine radicals and HF formation inside the chambers was conducted by analysing the exahust gas using the Ion Selective Electrode (ISE) method [7] by letting the return line of the gas bubble into a TISAB II solution and using the electrodes to read the $\mathrm{F}^{-}$concentration. The flow rate was kept constant while the ABS filters were changed. Figure 2 (b) shows that the rate of $F^{-}$production for the ECOGAS mixture is about two times higher than the Standard Gas Mixture when the currents have the same value, indicating that the HFO molecule breaks more easily than the R-134a molecule when detectors are under irradiation.

\section{Aging test}

In this study, the long term performance of the detectors with the ecogas mixture under irradiation were evaluted. Figure 3 (a) shows the trend of the dark currents at $3 \mathrm{kV}^{2}$ for the majority of 


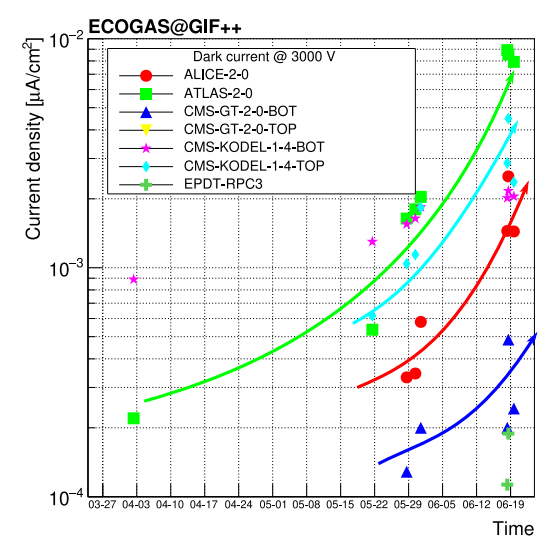

(a) Dark current of the RPCs during the irradiation period

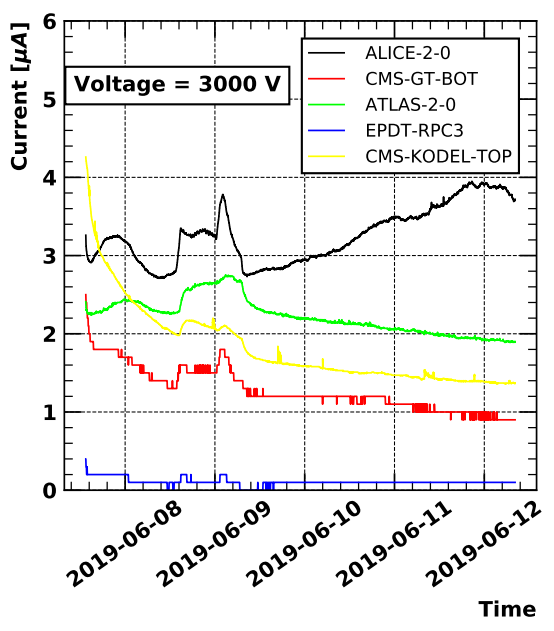

(b) Trend of the dark currents for the ECOGAS chambers

Figure 3: Irradiation campaign and recovery test performed on the ecogas chambers

the rpc chambers during the irradiation campaign. The plot reveals that there is an increase in dark currents of about 1-2 order of magnitudes after 30 days of irradiation. To confirm the correlation between the increase of the currents and the irradiation, chambers were lowered at a voltage of 3 $\mathrm{kV}$ so that the contribution of the photons was negligible. A decreasing trend in the currents could be observed for most of the chambers as shown if Figure 3 (b). An hypothesis an increase of the flow could help mitigate the accumulation of impurities at surface level of the chambers, lowering the absorbed currents.

\section{Conclusions}

The search for alternatives to R-134a for RPC detectors used at LHC experiments is a challenge as the gas mixture should be compatible with the electronics systems and should not induce a regression in the performance of the detectors. HFO has a low GWP and could be suitable for the RPCs if operated together with the addition of $\mathrm{CO}_{2}$ to lower the working point. Preliminary studies on the aging effects of the HFO-based gas mixtures were started and the long-term effects on the RPC detectors are currently being evaluated. The effects of the radiation on HFO resulted in a higher production of fluorine radicals and HF inside the gaps with respect to the R-134a. The RPCs performance were studied at different irradiation conditions and the aging test on the chambers was started. An increase of the dark currents was observed during the irradiation campaign. A recovery test was made by operating the detectors at low voltages and by flushing the chambers at higher flow rates. A decrease in the currents was observed: this seems to indicate that the detectors should be operated at higher flow in order to reduce impurities accumulation inside the chambers. Studies on different compositions of the gas mixture together with different flow conditions of the chambers have to be studied as they could improve the aging effects.

\footnotetext{
${ }^{2}$ The value of $3 \mathrm{kV}$ was chosen as a reference value of the ohmic currents in the present study.
} 


\section{References}

[1] R. Santonico and R. Cardarelli, Development of resistive plate counters, Nuclear Instruments and Methods in Physics Research 187 (1981) 377.

[2] M. Capeans, R. Guida and B. Mandelli, Strategies for reducing the environmental impact of gaseous detector operation at the cern lhc experiments, Nuclear Instruments and Methods in Physics Research Section A: Accelerators, Spectrometers, Detectors and Associated Equipment 845 (2017) 253.

[3] M. Schulz and D. Kourkoulas, Regulation (eu) no 517/2014 of the european parliament and of the council of 16 april 2014 on fluorinated greenhouse gases and repealing regulation (ec) no 842/2006, Off. J. Eur. Union 2014 (2014) L150.

[4] V. Peskov, M. Abbrescia and P. Fonte, Resistive Gaseous Detectors: Designs, Performance, and Perspectives. Wiley, 04, 2018.

[5] EN, EP, AIDA GIF++ collaboration, GIF++: A new CERN Irradiation Facility to test large-area detectors for the HL-LHC program, PoS ICHEP2016 (2016) 260.

[6] R. Arnaldi, A. Baldit, V. Barret, N. Bastid, G. Blanchard, E. Chiavassa et al., Influence of temperature and humidity on bakelite resistivity, Nuclear Instruments and Methods in Physics Research Section A: Accelerators, Spectrometers, Detectors and Associated Equipment 456 (2000) 140 .

[7] M. Abbrescia, A. Colaleo, R. Guida, G. Iaselli, F. Loddo, M. Maggi et al., Results about hf production and bakelite analysis for the cms resistive plate chambers, Nuclear Instruments and Methods in Physics Research Section A: Accelerators, Spectrometers, Detectors and Associated Equipment 594 (2008) 140 . 